\title{
Наблюдение областей отрицательной дифференциальной проводимости и генерации тока при туннелировании через нульмерные уровни дефектов барьера $h$-BN В гетероструктурах графен/ $h$-BN/графен
}

\author{
() Ю.Н. Ханин ${ }^{1}$, Е.Е. Вдовин ${ }^{1, \uparrow, ~ А . ~ М и щ е н к о ~}{ }^{2}$, К.С. Новоселов ${ }^{2}$ \\ ${ }^{1}$ Институт проблем технологии микроэлектроники и особо чистых материалов Российской академии наук, \\ 142432 Черноголовка, Московская область, Россия \\ ${ }^{2}$ School of Physics and Astronomy, University of Manchester, \\ Oxford Road, Manchester, M13 9PL, UK \\ I E-mail: vdov62@yandex.ru
}

Поступила в Редакцию 6 марта 2019 г.

В окончательной редакции 10 марта 2019 г.

Принята к публикации 13 марта 2019 г.

Исследовались туннелирование и магнитотуннелирование в ван-дер-ваальсовых гетеросистемах графен/ $h$-BN/графен, обнаружившие два новых типа систем, в которых области отрицательной дифференциальной проводимости реализуются в результате процессов резонансного туннелирования через уровни дефектов в барьере $h$-BN, а также происходит генерация тока, обусловленная их наличием.

Ключевые слова: туннелирование, магнитотуннелирование, ван-дер-ваальсовы гетеросистемы, графен.

DOI: $10.21883 /$ FTP.2019.08.47995.9093

Наличие областей отрицательной дифференциальной проводимости (ОДП) на вольт-амперных характеристиках $(\mathrm{BAX})$ в различных системах, проявляющихся как уменьшение тока с увеличением напряжения, привлекает постоянное внимание исследователей вледствие потребности в различных практических приборных приложениях наноэлектроники, таких как СВЧ генераторы, вентили и элементы для реализации систем квантовых вычислений. В современных ван-дер-ваальсовых графеновых гетеросистемах эти исследования еще более актуальны [1], поскольку в них предполагается возможность достижения частот генерации вплоть до терагерцового диапазона [2] и недавно уже показана возможность реализации областей ОДП. Так, например, при туннелировании и магнитотуннелировании через барьер из гексагонального нитрида бора $(h$-BN) между графеновыми слоями, кристаллические решетки которых хорошо сориентированы по углу, наблюдались как область ОДП, так и обусловленный этим эффект генерации тока $[3,4]$.

В данной работе исследовались туннелирование и магнитотуннелирование в ван-дер-ваальсовых гетеросистемах графен/h-BN/графен, обнаружившие две новые возможности реализации резких ОДП, связанные с наличием уровней дефектов в барьере $h$-BN.

Исследовавшиеся образцы представляли собой вертикальные ван-дер-ваальсовы гетероструктуры, получаемые путем механического расслоения и переноса слоев графена и $h$-BN (см. вставку на рис. 1,a). Изучалось два типа образцов, различавшихся верхним графеновым слоем и устройством нижнего затвора в первом типе верхний электрод представлял собой двухслойный графен, а затвор состоял из высоколе- гированного $\mathrm{Si}$ и диэлектрических слоев толщиной $D=290 \mathrm{Hм}\left(\mathrm{SiO}_{2}\right)+30 \mathrm{нм}(h-\mathrm{BN})$, а во втором - верхний электрод был из однослойного графена, а затвор состоял из графена электрода и подзатворного слоя $h$-BN толщиной $D=25-30$ нм, что обусловливало разные рабочие интервалы затворных напряжений $V_{\text {gate }}$. Контакты к слоям графена и верхняя затворная металлизация были изготовлены с помощью электронной литографии и напыления $\mathrm{AuGe} / \mathrm{Ni} / \mathrm{Au}$. Проводящие графеновые слои $\mathrm{Gr}_{b}$ и $\mathrm{Gr}_{t}$ были изготовлены в виде крестообразной структуры, разделенной в месте пересечения туннельным барьером $h$-BN толщиной $d$ в несколько монослоев (3-6 ML), площадь пересечения графеновых электродов была 25-50 мкм². Измерения туннельной проводимости/тока проводились четырехзондовым методом.

Схематическое изображение основных слоев первой структуры и измерительной цепи приведено на вставке к рис. 1,a. Несколько атомных слоев $h$-BN образуют туннельный барьер, расположенный между верхним двухслойным и нижним однослойным графеновыми электродами, BL Gr и SL Gr соответственно, которые действуют как электроды истока и стока. При приложении напряжения смещения, $V_{\text {bias, }}$ между ними химические потенциалы в верхнем и нижнем электродах $\left(\mu_{t}\right.$ и $\left.\mu_{b}\right)$ смещаются друг относительно друга и возникает экспоненциально растущий туннельный ток $I$ через барьер как результат переходов носителей из заполненных состояний в одном графеновом электроде в пустые состояния в другом. В случае, если в барьерном слое находится локализованное состояние любой природы и его энергетический уровень, двигаясь с изменением $V_{\text {bias, }}$ совпадает с химическим потенциалом $\mu_{t}$ в верхнем 
контакте, открывается канал резонансного туннелирования через это состояние, проявляющийся в токе в виде ступени (например, ступень $E_{1}$ на рис. $\left.1, a\right)$ или пика в дифференциальной проводимости, поскольку при дальнейшем увеличения $V_{\text {bias }}$ после порога этого резонанса ток через него меняется относительно слабо [5,6]. Если в разных слоях барьера находятся два близколежащих (в направлении, перпендикулярном туннелированию) состояния с несильно различающимися энергиями, то в случае совпадения их энергий на ВАХ будет наблюдаться резкий пик тока [7], как, например, на рис. 1, $a$. В наших образцах кристаллические углы $>10^{\circ}$. Такая разориентация подавляет прямое межзонное резонансное туннелирование с сохранением продольной слоям компоненты импульса и позволяет детально изучить малый туннельный ток при переходах через индивидуальное локализованное состояние [8]. Нижний затворный электрод, расположенный на подложке, используется
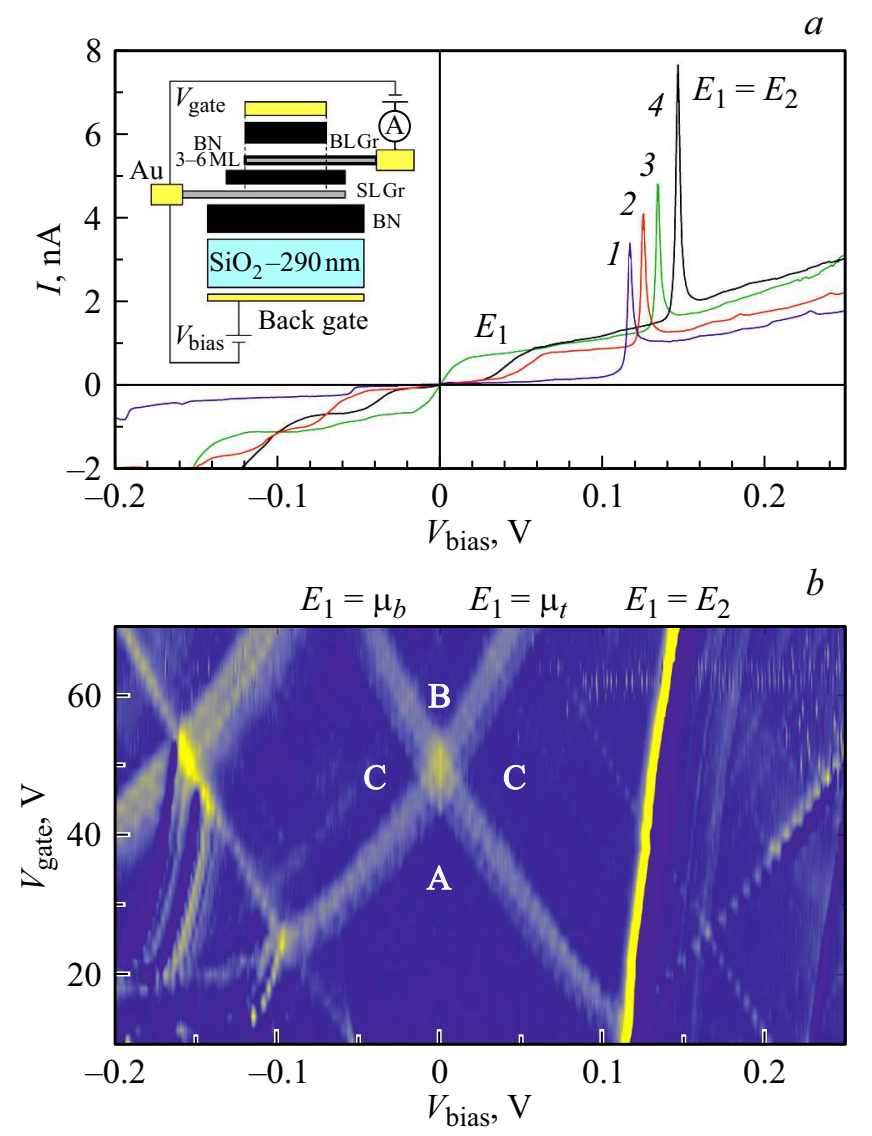

Рис. 1. Эспериментальные BAX $I\left(V_{\text {bias }}\right)(a)$ и карта проводимости в координатах $\left(V_{\text {gate }}, V_{\text {bias }}\right)(b)$ образца с верхним двухслойным графеновым электродом, измеренные при $T=4.2 \mathrm{~K}$. $a$ : ступеньки и пики в токе $E_{1}$ и $E_{1}=E_{2}$ отражают туннельные переходы между истоком и стоком через один или два уровня дефектов в барьере; $V_{\text {gate }}=10(1), 30$ (2), 50 (3) и 70 В (4). Описание линий и характерных зон на карте даны в тексте. На вставке - схематическое изображение основных слоев структуры и измерительной цепи.

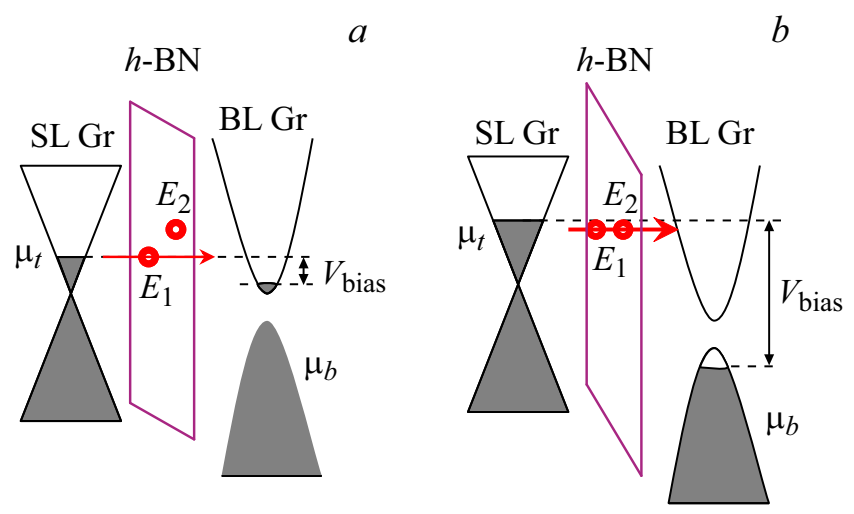

Рис. 2. Схемы туннельных переходов в образце первого типа с участием одного $(a)$ и двух $(b)$ уровней дефектов в барьере.

для регулировки концентрации носителей в графеновых слоях путем изменения напряжения затвора, $V_{\text {gate. }}$

Экспериментальные BAX образца с верхним двухслойным графеновым электродом, измеренные при температуре $T=4.2 \mathrm{~K}$, показаны на рис. $1, a$. Ступеньки и пики в токе, обозначенные $E_{1}$ и $E_{1}=E_{2}$, как уже отмечалось выше, обусловлены туннельными переходами между грефеновыми истоком и стоком через один или два уровня дефектов в барьере [5]. Движение ВАХ вызвано изменением затворного напряжения. Многократное увеличение резонансного тока при переходах через два уровня по сравнению с переходом через один дефект обусловлено естественным ростом туннельной прозрачности системы в такой ситуации. Наглядно туннельные процессы с участием одного или двух уровней дефектов $E_{1}$ и $E_{2}$ представлены на рис. 2 , где конусы и параболы соответствуют зонным структурам однослойного и двухслойного графена.

Наглядное представление о разных режимах транспорта в наших экспериментальных структурах дает рис. $1, b$, на котором показана карта проводимости в координатах $\left(V_{\text {gate }}, V_{\text {bias }}\right)$. Область А на карте соответствует малым значениям проводимости до начала порога резонансного туннелирования через состояние $E_{1}$ в барьере. Уровень $E_{1}$ в области А находится выше $\mu_{b}$ и $\mu_{t}$ одновременно, и туннельные переходы без изменения энергии невозможны из-за отсутствия в эмиттере носителей с необходимой энергией. При этом границы зоны А на карте соответствуют условию совпадения уровня $E_{1}$ с $\mu_{b}$ или $\mu_{t}$, т.е. $E_{1}=\mu_{b}$ и $E_{1}=\mu_{t}$ для положительных и отрицательных $V_{\text {bias }}$ соответственно. Область В на карте отличается от А только тем, что до порога резонанса $E_{1}$ находится ниже и $\mu_{b}$, и $\mu_{t}$, туннелирование невозможно из-за отсутствия свободных состояний для туннельных переходов в коллекторе. Точка пересечения линий, разграничивающих области А, В и С, соответствует условию, когда $E_{1}$ совпадает с $\mu_{b}$ и $\mu_{t}$ при $V_{\text {bias }}=0$. Области C на карте отражают мало меняющиеся значения тока после порога тунне- 
лирования через $E_{1}$. Самая яркая линия, обозначенная $E_{1}=E_{2}$, порождена переходами через пару дефектов, схематически показанными на рис. 2, $b$. Как видно из положения этой линии на карте, увеличение напряжения на затворе незначительно смещает условия совпадения уровней $E_{1}=E_{2}$ в сторону больших $V_{\text {bias }}$ (см. также BAX на рис. 1, $a$ при $V_{\text {gate }}=10,30,50$ и 70 В) из-за перераспределения потенциалов в барьерной области с изменением концентраций в стоке и истоке.

На этой структуре также наблюдались области ОДП при приложении магнитного поля. Магнитное поле, перпендикулярное графеновым слоям, приводило в такой ситуации к квантованию движения электронов в плоскости слоев и формированию неэквидистантных дискретных спектров уровней Ландау в однослойном графене и эквидистантных в двухслойном. Наблюдаемая при $B=0$ ступенька тока, соответствующая порогу туннелирования через уровень состояния дефекта (например, обсуждавшийся выше $E_{1}$ на рис. $\left.1, a\right)$, в магнитном поле превращалась в резкий пик тока с последующей областью отрицательной дифференциальной проводимости. Такой вид зависимости в магнитном поле обусловлен одновременным совпадением уровня Ландау в двух графеновых электродах и уровня дефекта $E_{1}$ в барьере. По сути такой резонансный канал $E_{1}$ в магнитном поле является одновременно усилителем туннельного тока и узким сканером плотностей состояний, дающих вклад в суммарный ток. На рис. 3, $a$ показаны две ВАХ при напряжении на затворе 51 (кривая 1) и 59 В (кривая 2), соответствующие туннелированию через уровень $E_{1}$ и различные уровни Ландау в магнитном поле $B=7$ Тл при температуре $4.2 \mathrm{~K}$. Карта проводимости, наглядно представляющая процессы магнитотуннелирования в этом образце при $B=7$ Тл и $T=4.2 \mathrm{~K}$, показана на рис. 3, $b$. Как видно из рисунка, плавная кривая движения порога резонанса $E_{1}=\mu_{t}$ превратилась в ступенчатую зависимость, где каждая вертикальная часть ступени отражает медленное изменение химического потенциала $\mu_{b}$ с изменением параметров $V_{\text {bias}}, V_{\text {gate }}$ в интервалах одновременного совпадения пары уровней Ландау в слоях и уровня дефекта, а горизонтальные соответствуют резкому изменению $V_{\text {bias }}$ в процессе перескока $\mu_{b}$ к следующему уровню Ландау, вызванную этим перестройку потенциалов в барьерной области и, как результат, переход к новым условиям резонансного туннелирования. Штриховые линии соответствуют ВАХ, представленным на рис. 3, $a$.

Аналогичный эффект ОДП при туннелировании между однослойными графеновыми электродами в образце второго типа в магнитном поле также был нами зарегистрирован (см. рис. 4,a). Из-за меньшей толщины барьерного слоя $h$-BN и центрального положения дефектного уровня в барьере [9] величина магнитотуннельного резонанса почти на 2 порядка превышала наблюдавшуюся в образце первого типа. Именно такая большая величина резонансного тока позволила нам, кроме того, зарегистрировать на этом образце генерацию тока в
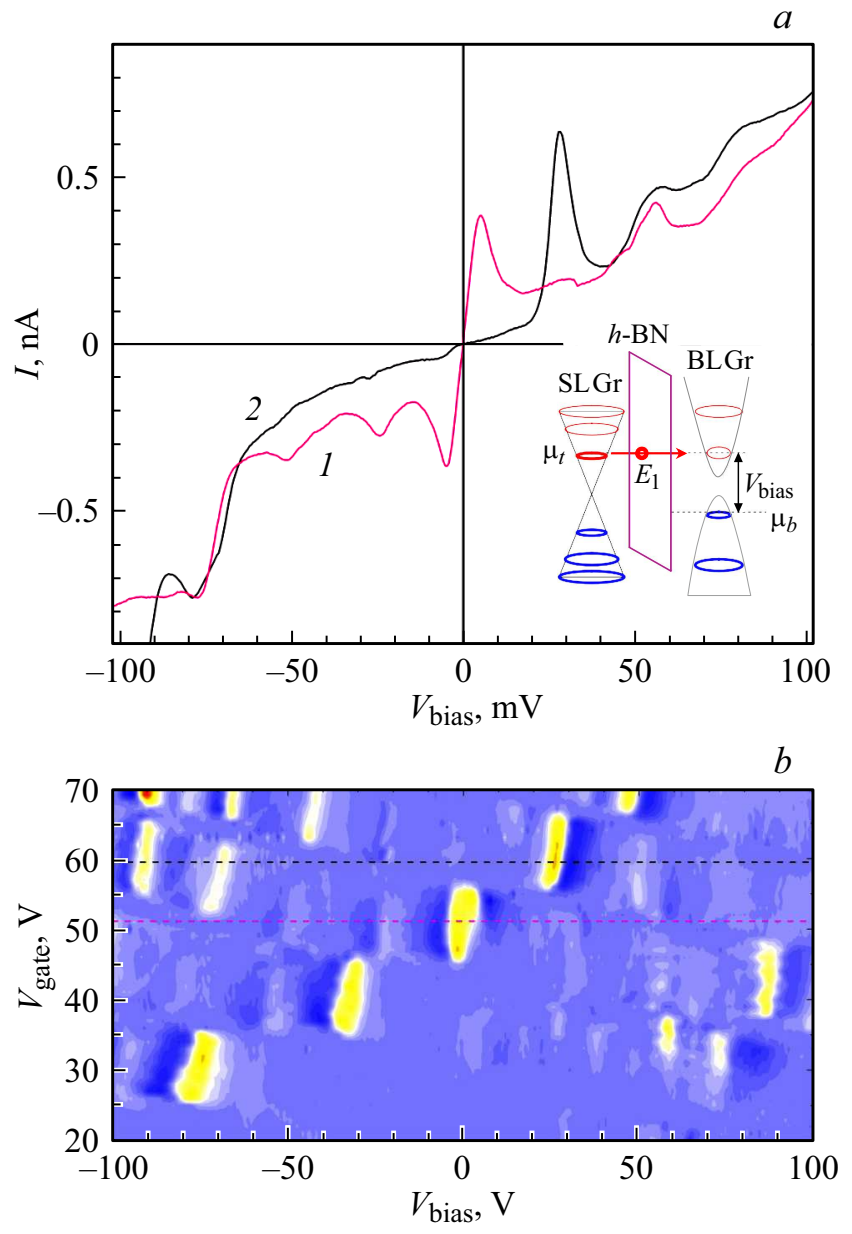

Рис. 3. Эспериментальные $\mathrm{BAX} I\left(V_{\text {bias }}\right)(a)$ и карта проводимости образца первого типа $(b)$ при $T=4.2 \mathrm{~K}$ и $B=7$ Тл. $a: V_{\text {gate }}=51$ (1) и $59 \mathrm{~B}(2)$. На вставке - схема магнитотуннельных переходов через состояние $E_{1}$. Описание деталей карты дано в тексте.

ОДП (см. рис. 4,b). Частота генерации составляла 700 Гц, что, вероятнее всего, обусловлено внутренними параметрами гетероструктуры, поскольку эта частота радикально отличается от предсказанной теоретически для подобных структур величины $\sim 50$ ГГц [23]. Такая генерация при магнитотуннелировании через уровень нульмерного состояния ранее не наблюдалась ни в графеновых, ни в полупроводниковых структурах. Для улучшения частотных характеристик систем, подобных нашим, требуется дополнительное исследование по их оптимизации, как, например, изготовление подобных образцов с существенно меньшими емкостями активных областей структуры, а также по оптимизации измерительной схемы. Однако эта трудоемкая задача не является предметом данной публикации. Вследствие более простой зонной структуры и симметричной структуры уровней Ландау в электродах образца второго типа (см. вставку к рис. 4,a), здесь достаточно просто определить конкретные пары уровней Ландау, участвующих в туннельном переходе через дефектный уровень. Так, 

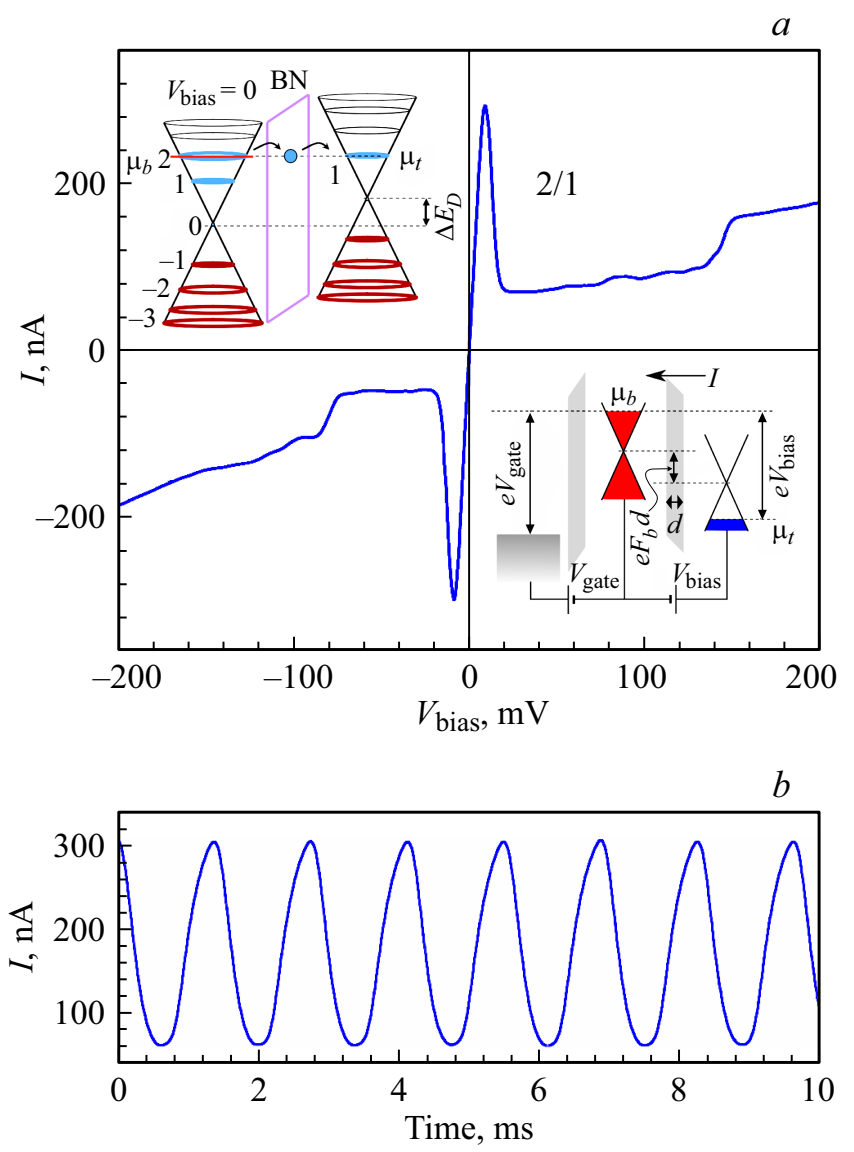

Рис. 4. $a-$ ВАХ образца второго типа в области туннельного перехода через уровень дефекта в перпендикулярном слоям магнитном поле $B=4$ Тл, $T=4.2 \mathrm{~K}$; на вставках: вверху схема магнитотуннельных переходов при $B=4$ Тл, $V_{\text {bias }}=0$, $\Delta E_{\mathrm{D}}$ - энергетический сдвиг точек Дирака в слоях; внизу - схематическое изображение основных слоев структуры и измерительной цепи, где $d-$ толщина барьера, $F_{b}-$ электрическое поле в барьере, $I-$ туннельный ток. $b-$ генерация туннельного тока в области резонанса.

например, резонансный пик, представленный на рис. 4, $a$, порожден туннельным переходом в магнитном поле 4 Тл через уровни Ландау с номерами 2 и 1 в нижнем и верхнем слоях графена соответственно. Отметим, что в образцах первого типа нам не удалось обнаружить генерации тока из-за существенно меньшей величины эффекта, что в свою очередь требовало проведения измерений с большими постоянными времени измерительного усилителя. Однако наличие ярких областей ОДП и в этом типе образцов указывает на принципиальную возможность генерации при условии оптимизации эксперимента.

Хотя рассмотренные в статье образцы, очевидно, не могут быть использованы для практических приложений ввиду случайности появления локализованных состояний внутри барьера, сам принцип использования нульмерных состояний в ван-дер-ваальсовых гетероструктурах представляется интересным, и уже осуществлена первая попытка создания таких структур со слоем выращенных в барьере $h$-BN графеновых квантовых точек [10].

\section{Финансирование работы}

Работа выполнена Ю.Н. Ханиным в рамках государственного задания 075-00475-19-00. Е.Е. Вдовин благодарит за финансовую поддержку Российский фонд фундаментальных исследований (грант 18-02-00425).

\section{Конфликт интересов}

Авторы заявляют, что у них нет конфликта интересов.

\section{Список литературы}

[1] S. Kang, X. Mou, B. Fallahazad, N. Prasad, X. Wu, A. Valsaraj, H.C.P. Movva, K. Kim, E. Tutuc, L.F. Register, S.K. Banerjee. J. Phys. D: Appl. Phys., 50, 383002 (2017).

[2] J. Gaskell, L. Eaves, K.S. Novoselov, A. Mishchenko, A.K. Geim, T.M. Fromhold, M.T. Greenaway. Appl. Phys. Lett., 107, 103105 (2015).

[3] A. Mishchenko, J.S. Tu, Y. Cao, R.V. Gorbachev, J.R. Wallbank, M.T. Greenaway, V.E. Morozov, S.V. Morozov, M.J. Zhu, S.L. Wong, F. Withers, C.R. Woods, Y.-J. Kim, K. Watanabe, T. Taniguchi, E.E. Vdovin, O. Makarovsky, T.M. Fromhold, V.I. Fal'ko, A.K. Geim, L. Eaves, K.S. Novoselov. Nature Nanotechnology, 9, 808 (2014).

[4] Ю.Н. Ханин, Е.Е. Вдовин, А. Мищенко, Ж.С. Ту, А. Козиков, Р.В. Горбачев, К.С. Новоселов. Письма ЖЭТФ, 104 (5), 342 (2016).

[5] M.T. Greenaway, E.E. Vdovin, D. Ghazaryan, A. Misra, A. Mishchenko, Y. Cao, Z. Wang, J.R. Wallbank, M. Holwill, Yu.N. Khanin, S.V. Morozov, K. Watanabe, T. Taniguchi, O. Makarovsky, T.M. Fromhold, A. Patanè, A.K. Geim, V.I. Fal'ko, K.S. Novoselov, L. Eaves. Commun. Phys., 1, Article number: 94 (2018).

[6] M.R. Deshpande, J.W. Sleight, M.A. Reed, R.G. Wheeler, R.J. Matyi. Phys. Rev. Lett., 76, 1328 (1996).

[7] T. Bryllert, M. Borgstrom, L.-E. Wernersson, W. Seifert, L. Samuelson. Appl. Phys. Lett., 82, 2655 (2003).

[8] Е.Е. Вдовин, Ю.Н. Ханин. Письма ЖЭТФ, 108(9), 674 (2018).

[9] B. Ricco, M.Ya. Azbel. Phys. Rev. B, 29, 1970 ( 1984).

[10] G. Kim, S. Kim, J. Jeon, S. Yoon, S. Hong, Y.J. Cho, A. Misra, S. Ozdemir, J. Yin, D. Ghazaryan, M. Holwill, A. Mishchenko, D.V. Andreeva, Y. Kim, H.Y. Jeong, A. Jang, H. Chung, A.K. Geim, K.S. Novoselov, B. Sohn, H.S. Shin. Nature Commun., 10, 230 (2019).

Редактор Л.В. Шаронова 


\section{Negative differential conductance and current generation at tunneling through zero-dimensional defect levels of the $h$-BN barrier in graphene/ $h$-BN/graphene heterostructures}

Yu.N. Khanin ${ }^{1}$, E.E. Vdovin ${ }^{1}$, A. Mishchenko², K.S. Novoselov ${ }^{2}$

${ }^{1}$ Institute of Problems of Microelectronics Technology and High Purity Materials,

Russian Academy of Sciences,

142432 Chernogolovka, Moscow Region, Russia

2 School of Physics and Astronomy,

University of Manchester,

Oxford Road, Manchester, M13 9PL, UK

Abstract In this work, we studied tunneling and magnetotunneling in van der Waals heterosystems graphene/ $h$-BN/graphene, which revealed two new types of systems in which the areas of negative differential conductivity are realized as a result of resonant tunneling processes through the levels of defects in the $h$-BN barrier, as well as current generation due to their presence. 